\title{
Tumor Regression Grade
}

National Cancer Institute

\section{Source}

National Cancer Institute. Tumor Regression Grade. NCI Thesaurus. Code C106304.

A pathologic grading system used to evaluate the tumor response to preoperative

chemotherapy and radiation. 